DOI 10.4171/JEMS/700

Hanspeter Kraft · Andriy Regeta

\title{
Automorphisms of the Lie algebra of vector fields on affine $n$-space
}

Received July 27, 2014

Abstract. We study the vector fields $\operatorname{Vec}\left(\mathbb{A}^{n}\right)$ on affine $n$-space $\mathbb{A}^{n}$, the subspace $\operatorname{Vec}^{c}\left(\mathbb{A}^{n}\right)$ of vector fields with constant divergence, and the subspace $\operatorname{Vec}^{0}\left(\mathbb{A}^{n}\right)$ of vector fields with divergence zero, and we show that their automorphisms, as Lie algebras, are induced by the automorphisms of $\mathbb{A}^{n}$ :

$$
\operatorname{Aut}\left(\mathbb{A}^{n}\right) \stackrel{\sim}{\rightarrow} \operatorname{Aut}_{\mathrm{Lie}}\left(\operatorname{Vec}\left(\mathbb{A}^{n}\right)\right) \stackrel{\sim}{\rightarrow} \operatorname{Aut}_{\mathrm{Lie}}\left(\operatorname{Vec}^{c}\left(\mathbb{A}^{n}\right)\right) \stackrel{\sim}{\rightarrow} \operatorname{Aut}_{\mathrm{Lie}}\left(\operatorname{Vec}^{0}\left(\mathbb{A}^{n}\right)\right)
$$

This generalizes results of the second author obtained in dimension 2 [Reg13]. The case of $\operatorname{Vec}\left(\mathbb{A}^{n}\right)$ goes back to Kulikov [Ku192].

This generalization is crucial in the context of infinite-dimensional algebraic groups, because $\operatorname{Vec}^{c}\left(\mathbb{A}^{n}\right)$ is canonically isomorphic to the Lie algebra of $\operatorname{Aut}\left(\mathbb{A}^{n}\right)$, and $\operatorname{Vec}^{0}\left(\mathbb{A}^{n}\right)$ is isomorphic to the Lie algebra of the closed subgroup $\operatorname{SAut}\left(\mathbb{A}^{n}\right) \subset \operatorname{Aut}\left(\mathbb{A}^{n}\right)$ of automorphisms with Jacobian determinant equal to 1 .

Keywords. Automorphisms, vector fields, Lie algebras, affine $n$-space

\section{Introduction}

Let $K$ be an algebraically closed field of characteristic zero. Denote by $\operatorname{Vec}\left(\mathbb{A}^{n}\right)$ the Lie algebra of polynomial vector fields on affine $n$-space $\mathbb{A}^{n}=K^{n}$ :

$$
\operatorname{Vec}\left(\mathbb{A}^{n}\right)=\operatorname{Der}\left(K\left[x_{1}, \ldots, x_{n}\right]\right)=\left\{\sum_{i} f_{i} \frac{\partial}{\partial x_{i}} \mid f_{i} \in K\left[x_{1}, \ldots, x_{n}\right]\right\}
$$

where we use the standard identification of a derivation $\delta$ with $\sum_{i} \delta\left(x_{i}\right) \frac{\partial}{\partial x_{i}}$. The group $\operatorname{Aut}\left(\mathbb{A}^{n}\right)$ of polynomial automorphisms of $\mathbb{A}^{n}$ acts on $\operatorname{Vec}\left(\mathbb{A}^{n}\right)$ in the usual way. For $\varphi \in \operatorname{Aut}\left(\mathbb{A}^{n}\right)$ and $\delta \in \operatorname{Vec}\left(\mathbb{A}^{n}\right)=\operatorname{Der}\left(K\left[x_{1}, \ldots, x_{n}\right]\right)$ we define

$$
\operatorname{Ad}(\varphi) \delta:=\varphi^{*-1} \circ \delta \circ \varphi^{*}
$$

H. Kraft: Universität Basel, Departement Mathematik und Informatik, Spiegelgasse 1, CH-4051 Basel, Switzerland; e-mail: hanspeter.kraft@ unibas.ch

A. Regeta: Université de Grenoble I, Institut Fourier, UMR 5582 CNRS-UJF, BP 74, F-38402 St. Martin d'Heres Cedex, France; e-mail: andriyregeta@ gmail.com

Mathematics Subject Classification (2010): Primary 17B66; Secondary 22F50 
where $\varphi^{*}: K\left[x_{1}, \ldots, x_{n}\right] \rightarrow K\left[x_{1}, \ldots, x_{n}\right], f \mapsto f \circ \varphi$, is the comorphism of $\varphi$. It is shown in [Ku192] that $\operatorname{Ad}: \operatorname{Aut}\left(\mathbb{A}^{n}\right) \rightarrow \operatorname{Aut}_{\text {Lie }}\left(\operatorname{Vec}\left(\mathbb{A}^{n}\right)\right)$ is an isomorphism. We will give a short proof in Section 3.

Recall that the divergence of a vector field $\delta=\sum_{i} f_{i} \frac{\partial}{\partial x_{i}}$ is defined by Div $\delta:=$ $\sum_{i} \frac{\partial f_{i}}{\partial x_{i}}$. This leads to the following subspaces of $\operatorname{Vec}\left(\mathbb{A}^{n}\right)$ :

$$
\operatorname{Vec}^{0}\left(\mathbb{A}^{n}\right):=\left\{\delta \in \operatorname{Vec}\left(\mathbb{A}^{n}\right) \mid \operatorname{Div} \delta=0\right\} \subset \operatorname{Vec}{ }^{c}\left(\mathbb{A}^{n}\right):=\left\{\delta \in \operatorname{Vec}\left(\mathbb{A}^{n}\right) \mid \operatorname{Div} \delta \in K\right\},
$$

which are Lie subalgebras, because $\operatorname{Div}[\delta, \eta]=\delta(\operatorname{Div} \eta)-\eta(\operatorname{Div} \delta)$. We have

$$
\operatorname{Vec}^{c}\left(\mathbb{A}^{n}\right)=\operatorname{Vec}^{0}\left(\mathbb{A}^{n}\right) \oplus K \partial_{E} \quad \text { where } \quad \partial_{E}:=\sum_{i} x_{i} \frac{\partial}{\partial x_{i}} \text { is the Euler field. }
$$

The aim of this note is to prove the following result about the automorphism groups of these Lie algebras.

Main Theorem. There are canonical isomorphisms

$$
\operatorname{Aut}\left(\mathbb{A}^{n}\right) \stackrel{\sim}{\rightarrow} \operatorname{Aut}_{\text {Lie }}\left(\operatorname{Vec}\left(\mathbb{A}^{n}\right)\right) \stackrel{\sim}{\rightarrow} \operatorname{Aut}_{\mathrm{Lie}}\left(\operatorname{Vec}^{c}\left(\mathbb{A}^{n}\right)\right) \stackrel{\sim}{\rightarrow} \operatorname{Aut}_{\mathrm{Lie}}\left(\operatorname{Vec}^{0}\left(\mathbb{A}^{n}\right)\right) .
$$

Remark 1.1. It is easy to see that the theorem holds for any field $K$ of characteristic zero. In fact, all the homomorphisms are defined over $\mathbb{Q}$, and are equivariant with respect to the obvious actions of the Galois group $\Gamma=\operatorname{Gal}(\bar{K} / K)$.

As a consequence, we will get the next result (see Corollary 4.4) which goes back to Kulikov [Ku192, Theorem 4].

Corollary. If every injective endomorphism of the Lie algebra $\operatorname{Vec}\left(\mathbb{A}^{n}\right)$ is an automorphism, then the Jacobian Conjecture holds in dimension $n$.

Remark 1.2. The Main Theorem has another interesting consequence. The group $\operatorname{Aut}\left(\mathbb{A}^{n}\right)$ is an infinite-dimensional algebraic group in the sense of Shafarevich [Sha66, Sha81], briefly an ind-group (cf. [Kum02]), and its Lie algebra is canonically isomorphic to $\operatorname{Vec}^{c}\left(\mathbb{A}^{n}\right)$. It was recently shown by Belov-Kanel and Yu [BKY12] that every automorphism of $\operatorname{Aut}\left(\mathbb{A}^{n}\right)$ as an ind-group is inner. Using the Main Theorem above one can give a new proof of this and extend it to the closed subgroup $\operatorname{SAut}\left(\mathbb{A}^{n}\right) \subset \operatorname{Aut}\left(\mathbb{A}^{n}\right)$ of automorphisms with Jacobian determinant equal to 1 . The details can be found in [Kra15] where we also show that the maps in the Main Theorem are isomorphisms of ind-groups.

We add here a lemma which will be used later on.

Lemma 1.3. $\operatorname{Vec}\left(\mathbb{A}^{n}\right)$ and $\operatorname{Vec}^{0}\left(\mathbb{A}^{n}\right)$ are simple Lie algebras, and

$$
\operatorname{Vec}^{0}\left(\mathbb{A}^{n}\right)=\left[\operatorname{Vec}^{c}\left(\mathbb{A}^{n}\right), \operatorname{Vec}^{c}\left(\mathbb{A}^{n}\right)\right]
$$

Proof. The formula $\left[\frac{\partial}{\partial x_{j}}, \sum_{i} f_{i} \frac{\partial}{\partial x_{i}}\right]=\sum_{i} \frac{\partial f_{i}}{\partial x_{j}} \frac{\partial}{\partial x_{i}}$ shows that every nonzero ideal $\mathfrak{a}$ of $\operatorname{Vec}\left(\mathbb{A}^{n}\right)$ contains a nonzero element from $\sum_{i} K \frac{\partial}{\partial x_{i}}$, and $\left[x_{\ell} \frac{\partial}{\partial x_{j}}, \frac{\partial}{\partial x_{i}}\right]=-\delta_{i \ell} \frac{\partial}{\partial x_{j}}$ implies that $\sum_{i} K \frac{\partial}{\partial x_{i}} \subseteq \mathfrak{a}$. Now we use $\left[f \frac{\partial}{\partial x_{j}}, \frac{\partial}{\partial x_{i}}\right]=-\frac{\partial f}{\partial x_{i}} \frac{\partial}{\partial x_{j}}$ to conclude that $\mathfrak{a}=\operatorname{Vec}\left(\mathbb{A}^{n}\right)$, hence $\operatorname{Vec}\left(\mathbb{A}^{n}\right)$ is simple. (See also [Jor78, Theorem, p. 446].)

The second statement is proved in a similar way and can be found in [Sha81, Lemma 3], and from that the last claim follows immediately. 


\section{Group actions and vector fields}

If an algebraic group $G$ acts on an affine variety $X$, we obtain a canonical linear map Lie $G \rightarrow \operatorname{Vec}(X)$ defined in the usual way (cf. [Kra11, II.4.4]). For every $A \in \operatorname{Lie} G$ the associated vector field $\xi_{A}$ on $X$ is defined by

$$
\left(\xi_{A}\right)_{x}:=d \mu_{x}(A) \quad \text { for } x \in X
$$

where $\mu_{x}: G \rightarrow X, g \mapsto g x$, is the orbit map in $x \in X$. It is well-known that the linear map $A \mapsto \xi_{A}$ is an anti-homomorphism of Lie algebras, and that its kernel is equal to the Lie algebra of the kernel of the action $G \rightarrow \operatorname{Aut}(X)$. In particular, for any algebraic subgroup $G \subset \operatorname{Aut}\left(\mathbb{A}^{n}\right)$ we have a canonical injection Lie $G \hookrightarrow \operatorname{Vec}\left(\mathbb{A}^{n}\right)$; we will denote the image by $L(G)$. Let us point out that a connected $G \subset \operatorname{Aut}\left(\mathbb{A}^{n}\right)$ is determined by $L(G)$, i.e., if $L(G)=L(H)$ for algebraic subgroups $G, H \subset \operatorname{Aut}\left(\mathbb{A}^{n}\right)$, then $G^{0}=H^{0}$.

Recall that the vector field $\delta \in \operatorname{Vec}\left(\mathbb{A}^{n}\right)$ is called locally nilpotent if the action of $\delta$ on $K\left[x_{1}, \ldots, x_{n}\right]$ is locally nilpotent, i.e., for any $f \in K\left[x_{1}, \ldots, x_{n}\right]$ we have $\delta^{m}(f)=0$ if $m$ is large enough. Every such $\delta$ defines an action of the additive group $K^{+}$on $\mathbb{A}^{n}$ such that $\delta=\xi_{1}$ where $1 \in K=$ Lie $K^{+}$(see (2.1) above).

Lemma 2.1. Let $\mathbf{u} \subset \operatorname{Vec}\left(\mathbb{A}^{n}\right)$ be a finite-dimensional commutative Lie subalgebra consisting of locally nilpotent vector fields. Then there is a commutative unipotent algebraic subgroup $U \subset \operatorname{Aut}\left(\mathbb{A}^{n}\right)$ such that $L(U)=\mathbf{u}$. If $\mathfrak{c e n t}_{\mathrm{Vec}\left(\mathbb{A}^{n}\right)}(\mathbf{u})=\mathbf{u}$, then $U$ acts transitively on $\mathbb{A}^{n}$.

Proof. It is clear that $\mathbf{u}=L(U)$ for a commutative unipotent subgroup $U \subset \operatorname{Aut}\left(\mathbb{A}^{n}\right)$. In fact, choose a basis $\left(\delta_{1}, \ldots, \delta_{m}\right)$ of $\mathbf{u}$ and consider the corresponding actions $\rho_{i}: K^{+} \rightarrow$ $\operatorname{Aut}\left(\mathbb{A}^{n}\right)$. Since the associated vector fields $\delta_{i}$ commute, the same holds for the actions $\rho_{i}$, so that we get an action of $\left(K^{+}\right)^{m}$. It follows that the image $U \subset \operatorname{Aut}\left(\mathbb{A}^{n}\right)$ is a commutative unipotent subgroup with $L(U)=\mathbf{u}$.

Assume that the action of $U$ is not transitive. Then all orbits have dimension $<n$, because orbits of unipotent groups acting on affine varieties are closed (see [Bor91, Chap. I, Proposition 4.10]). But then there is a nonconstant $U$-invariant function $f \in$ $K\left[x_{1}, \ldots, x_{n}\right]$. This implies that for every $\delta \in \mathbf{u}$ the vector field $f \delta$ commutes with $\mathbf{u}$ and thus belongs to $\mathfrak{c e n t}_{\operatorname{Vec}\left(\mathbb{A}^{n}\right)}(\mathbf{u})$, contradicting the assumption.

Any $\delta \in \operatorname{Vec}\left(\mathbb{A}^{n}\right)$ acts on the functions $K\left[x_{1}, \ldots, x_{n}\right]$ as a derivation, and on the Lie algebra $\operatorname{Vec}\left(\mathbb{A}^{n}\right)$ by the adjoint action, $\operatorname{ad}(\delta) \mu:=[\delta, \mu]=\delta \circ \mu-\mu \circ \delta$. These two actions are related as shown in the following lemma whose proof is obvious.

Lemma 2.2. Let $\delta, \mu \in \operatorname{Vec}\left(\mathbb{A}^{n}\right)$ be commuting vector fields and $f \in K\left[x_{1}, \ldots, x_{n}\right]$. Then

$$
\operatorname{ad}(\delta)(f \mu)=\delta(f) \mu .
$$

In particular, if $\operatorname{ad}(\delta)$ is locally nilpotent on $\operatorname{Vec}\left(\mathbb{A}^{n}\right)$, then $\delta$ is locally nilpotent as a vector field. 


\section{Proof of the Main Theorem, part I}

We first give a proof of the following result which goes back to Kulikov [Kul92, proof of Theorem 4]; see also [Bav13].

Theorem 3.1. The canonical map $\operatorname{Ad}: \operatorname{Aut}\left(\mathbb{A}^{n}\right) \rightarrow \operatorname{Aut}_{\text {Lie }}\left(\operatorname{Vec}\left(\mathbb{A}^{n}\right)\right)$ is an isomorphism.

Denote by $\operatorname{Aff}_{n} \subset \operatorname{Aut}\left(\mathbb{A}^{n}\right)$ the closed subgroup of affine transformations and by $S=$ $\left(K^{+}\right)^{n} \subset \operatorname{Aff}_{n}$ the subgroup of translations. Then

$$
L\left(\operatorname{Aff}_{n}\right)=\left\langle x_{i} \partial_{x_{j}}, \partial_{x_{k}} \mid 1 \leq i, j, k \leq n\right\rangle \supset L(S)=\left\langle\partial_{x_{1}}, \ldots, \partial_{x_{n}}\right\rangle
$$

where $\partial_{x_{j}}:=\partial / \partial x_{j}$. Set $\mathfrak{a f f} \mathfrak{f}_{n}:=\operatorname{Lie~Aff}_{n}$ and $\mathfrak{s a f f} \mathfrak{f}_{n}:=\left[\mathfrak{a f f} \mathfrak{f}_{n}, \mathfrak{a f f} \mathfrak{f}_{n}\right]=\operatorname{LieSAff}_{n}$ where $\operatorname{SAff}_{n}:=\left(\operatorname{Aff}_{n}, \operatorname{Aff}_{n}\right) \subset \operatorname{Aff}_{n}$ is the commutator subgroup, i.e. the closed subgroup of those affine transformations $x \mapsto g x+b$ where $g \in \mathrm{SL}_{n}$. The next lemma is certainly known. For the convenience of the reader we indicate a short proof.

Lemma 3.2. The canonical homomorphisms

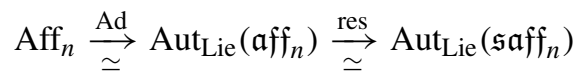

are isomorphisms.

Proof. It is clear that the homomorphisms

$$
\operatorname{Ad}: \operatorname{Aff}_{n} \rightarrow \operatorname{Aut}_{\text {Lie }}\left(\mathfrak{a f f} f_{n}\right) \text { and res: } \operatorname{Aut}_{\text {Lie }}\left(\mathfrak{a f f} \mathfrak{f}_{n}\right) \rightarrow \operatorname{Aut}_{\text {Lie }}\left(\mathfrak{s a f f} \mathfrak{f}_{n}\right)
$$

are both injective. Thus it suffices to show that the composition res $\circ$ Ad is surjective.

We write the elements of $\operatorname{Aff}_{n}$ in the form $(v, g)$ with $v \in S=\left(K^{+}\right)^{n}, g \in \mathrm{GL}_{n}$ where $(v, g) x=g x+v$ for $x \in \mathbb{A}^{n}$. It follows that $(v, g)(w, h)=(v+g w, g h)$. Similarly, $(a, A) \in \mathfrak{a f f}_{n}$ means that $a \in \mathfrak{s}:=\operatorname{Lie} S=K^{n}, A \in \mathfrak{g l}_{n}$, and $(a, A) x=A x+a$. For the adjoint representation of $g \in \mathrm{GL}_{n}$ and of $v \in S$ on $\mathfrak{a f f}_{n}$ we find

$$
\operatorname{Ad}(g)(a, A)=\left(g a, g A g^{-1}\right) \quad \text { and } \quad \operatorname{Ad}(v)(a, A)=(a-A v, A),
$$

and thus, for $(b, B) \in \mathfrak{a f f} \mathfrak{f}_{n}$,

$$
\operatorname{ad}(B)(a, A)=(B a,[B, A]) \quad \text { and } \operatorname{ad}(b)(a, A)=(a-A b, A) .
$$

Now let $\theta$ be an automorphism of the Lie algebra $\mathfrak{s a f f} \mathfrak{f}_{n}$. Then $\theta(\mathfrak{s})=\mathfrak{s}$ since $\mathfrak{s}$ is the solvable radical of $\mathfrak{s a f f} f_{n}$. Since $g:=\left.\theta\right|_{\mathfrak{s}} \in \mathrm{GL}_{n}$, we can replace $\theta$ by $\operatorname{Ad}\left(g^{-1}\right) \circ \theta$ and thus assume, by (3.2), that $\theta$ is the identity on $\mathfrak{s}$. This implies that $\theta(a, A)=(a+\ell(A), \bar{\theta}(A))$ where $\ell: \mathfrak{s l}_{n} \rightarrow \mathfrak{s}$ is a linear map and $\bar{\theta}: \mathfrak{s l}_{n} \stackrel{\sim}{\rightarrow} \mathfrak{s l}_{n}$ is a Lie algebra automorphism.

From (3.3) we $\operatorname{get} \operatorname{ad}(b, B)(a, 0)=\operatorname{ad}(B)(a, 0)=(B a, 0)$ for all $a \in \mathfrak{s}$, hence

$$
\begin{aligned}
(B a, 0)=\theta(B a, 0) & =\theta(\operatorname{ad}(B)(a, 0)) \\
& =\operatorname{ad}(\theta(B))(a, 0)=\operatorname{ad}(\bar{\theta}(B))(a, 0)=(\bar{\theta}(B) a, 0) .
\end{aligned}
$$


Thus $\bar{\theta}(B)=B$, i.e. $\theta(a, A)=(a+\ell(A), A)$. Now an easy calculation shows that $\ell([A, B])=A \ell(B)-B \ell(A)$. This means that $\ell$ is a cocycle of $\mathfrak{s l}_{n}$. Since $\mathfrak{s l}_{n}$ is semisimple, $\ell$ is a coboundary, and thus $\ell(A)=A v$ for a suitable $v \in K^{n}$. In view of (3.3) this implies that $\theta=\operatorname{Ad}(-v)$, and the claim follows.

Proof of Theorem 3.1. It is clear that the homomorphism

$$
\operatorname{Ad}: \operatorname{Aut}\left(\mathbb{A}^{n}\right) \rightarrow \operatorname{Aut}_{\text {Lie }}\left(\operatorname{Vec}\left(\mathbb{A}^{n}\right)\right)
$$

is injective. So let $\theta \in \operatorname{Aut}_{\text {Lie }}\left(\operatorname{Vec}\left(\mathbb{A}^{n}\right)\right)$ be an arbitrary automorphism.

We have seen above that $L(S)=\left\langle\partial_{x_{1}}, \ldots, \partial_{x_{n}}\right\rangle \subset \operatorname{Vec}\left(\mathbb{A}^{n}\right)$ where $S \subset \operatorname{Aff}_{n}$ is the subgroup of translations. Clearly, for every $\delta \in L(S)$ the adjoint $\operatorname{action} \operatorname{ad}(\delta)$ on $\operatorname{Vec}\left(\mathbb{A}^{n}\right)$ is locally nilpotent, and the same holds for any element from $\mathbf{u}:=\theta(L(S))$. It follows from Lemma 2.2 that $\mathbf{u}$ consists of locally nilpotent vector fields. Hence, by Lemma $2.1, \mathbf{u}=L(U)$ for a commutative unipotent subgroup $U$ of dimension $n$. Moreover, $\mathfrak{c e n t} t_{\operatorname{Vec}\left(\mathbb{A}^{n}\right)}(L(S))=L(S)$, and so $\mathfrak{c e n t} t_{\operatorname{Vec}\left(\mathbb{A}^{n}\right)}(\mathbf{u})=\mathbf{u}$, which implies, again by Lemma 2.1, that $U$ acts transitively on $\mathbb{A}^{n}$. Thus every orbit map $U \rightarrow \mathbb{A}^{n}$ is an isomorphism. It follows that there is an automorphism $\varphi \in \operatorname{Aut}\left(\mathbb{A}^{n}\right)$ such that $\varphi U \varphi^{-1}=S$. In fact, fix a group isomorphism $\psi: U \stackrel{\sim}{\rightarrow} S$ and take the orbit maps $\mu_{S}: S \stackrel{\sim}{\rightarrow} \mathbb{A}^{n}$ and $\mu_{U}: U \stackrel{\sim}{\rightarrow} \mathbb{A}^{n}$ at the origin $0 \in \mathbb{A}^{n}$. Then one easily sees that $\varphi:=\mu_{S} \circ \psi \circ \mu_{U}^{-1}$ has the property that $\varphi \circ u \circ \varphi^{-1}=\psi(u)$ for all $u \in U$.

It follows that the automorphism $\theta^{\prime}:=\operatorname{Ad}(\varphi) \circ \theta \in \operatorname{Aut}_{\text {Lie }}\left(\operatorname{Vec}\left(\mathbb{A}^{n}\right)\right)$ sends $L(S)$ isomorphically onto itself. Now the relations $\left[\partial_{x_{i}}, x_{j} \partial_{x_{k}}\right]=\delta_{i j} \partial_{x_{k}}$ imply that $\theta^{\prime}\left(L\left(\operatorname{Aff}_{n}\right)\right)=$ $L\left(\operatorname{Aff}_{n}\right)$. By Lemma 3.2, there is an $\alpha \in \operatorname{Aff}_{n}$ such that $\operatorname{Ad}(\alpha) \circ \theta^{\prime}$ is the identity on $L\left(\operatorname{Aff}_{n}\right)$. Hence, by the next lemma, $\operatorname{Ad}(\alpha) \circ \theta^{\prime}=\mathrm{id}$, because $\operatorname{Ad}(\lambda E)$ acts by multiplication with $\lambda$ on $L(S)$, and $\operatorname{so} \theta=\operatorname{Ad}\left(\varphi^{-1} \circ \alpha^{-1}\right)$.

Lemma 3.3. Let $\theta$ be an injective endomorphism of one of the Lie algebras $\operatorname{Vec}\left(\mathbb{A}^{n}\right)$, $\operatorname{Vec}^{c}\left(\mathbb{A}^{n}\right)$ or $\operatorname{Vec}^{0}\left(\mathbb{A}^{n}\right)$. If $\theta$ is the identity on $L\left(\mathrm{SL}_{n}\right)$, then $\theta=\operatorname{Ad}(\lambda E)$ for some $\lambda \in K^{*}$.

Proof. We consider the action of $\mathrm{GL}_{n}$ on $\operatorname{Vec}\left(\mathbb{A}^{n}\right)$. Denote by $\operatorname{Vec}\left(\mathbb{A}^{n}\right)_{d}$ the homogeneous vector fields of degree $d$, i.e.

$$
\operatorname{Vec}\left(\mathbb{A}^{n}\right)_{d}:=\bigoplus_{i} K\left[x_{1}, \ldots, x_{n}\right]_{d+1} \partial_{x_{i}} \simeq K\left[x_{1}, \ldots, x_{n}\right]_{d+1} \otimes K^{n}
$$

Note that $\lambda E \in \mathrm{GL}_{n}$ acts by scalar multiplication with $\lambda^{-d}$ on $\operatorname{Vec}\left(\mathbb{A}^{n}\right)_{d}$. We have split exact sequences of $\mathrm{GL}_{n}$-modules

$$
0 \rightarrow \operatorname{Vec}^{0}\left(\mathbb{A}^{n}\right)_{d} \rightarrow \operatorname{Vec}\left(\mathbb{A}^{n}\right)_{d} \stackrel{\text { Div }}{\longrightarrow} K\left[x_{1}, \ldots, x_{n}\right]_{d} \rightarrow 0
$$

where $K\left[x_{1}, \ldots, x_{n}\right]_{-1}=(0)$. Moreover, the $\mathrm{SL}_{n}$-modules $\operatorname{Vec}^{0}\left(\mathbb{A}^{n}\right)_{d}($ for $d \geq-1)$ and $K\left[x_{1}, \ldots, x_{n}\right]_{d}$ (for $d \geq 0$ ) are simple and pairwise nonisomorphic (see Pieri's formula [Pro07, Chap. 9, Section 10.2]). The splitting of (3.4) is given by $K\left[x_{1}, \ldots, x_{n}\right]_{d} \partial_{E} \subset$ $\operatorname{Vec}\left(\mathbb{A}^{n}\right)_{d}$ where $\partial_{E}=x_{1} \partial_{x_{1}}+\cdots+x_{n} \partial_{x_{n}}$ is the Euler field. In fact, the Euler field is fixed under $\mathrm{GL}_{n}$ and $\operatorname{Div}\left(f \partial_{E}\right)=(d+1) f$ for $f \in K\left[x_{1}, \ldots, x_{n}\right]_{d}$. 
Now let $\theta$ be an injective endomorphism of $\operatorname{Vec}\left(\mathbb{A}^{n}\right)$. If $\theta$ is the identity on $L\left(\mathrm{SL}_{n}\right)$, then $\theta$ is $\mathrm{SL}_{n}$-equivariant and thus acts as a scalar $\lambda_{d}$ on $\operatorname{Vec}^{0}\left(\mathbb{A}^{n}\right)_{d}$ and as a scalar $\mu_{d}$ on $K\left[x_{1}, \ldots, x_{n}\right]_{d} \partial_{E}$, by Schur's Lemma. The relations

$$
\left[x_{j}^{e+1} \partial_{x_{i}}, x_{i}^{d+1} \partial_{x_{j}}\right]=(d+1) x_{i}^{d} x_{j}^{e+1} \partial_{x_{j}}-(e+1) x_{i}^{d+1} x_{j}^{e} \partial_{x_{i}}, \quad i \neq j,
$$

show that $\lambda_{e} \lambda_{d}=\lambda_{e+d}$, hence $\lambda_{d}=\lambda^{d}$ for $\lambda:=\lambda_{1}$. The relations

$$
\left[x_{i}^{e} \partial_{E}, x_{i}^{d} \partial_{E}\right]=(d-e) x_{i}^{e+d} \partial_{E}
$$

show that $\mu_{e} \mu_{d}=\mu_{e+d}$ for $e \neq d$, which also implies that $\mu_{d}=\mu^{d}$ for $\mu:=\mu_{1}$. Finally, from the relation $\left[\partial_{x_{1}}, x_{2} \partial_{E}\right]=x_{2} \partial_{x_{1}}$, we get $\lambda=\mu$, and so $\theta=\operatorname{Ad}\left(\lambda^{-1} \mathrm{id}\right)$. This proves the claim for $\operatorname{Vec}\left(\mathbb{A}^{n}\right)$. The other two cases follow along the same lines.

\section{4. Étale morphisms and vector fields}

In the first section we defined the action of $\operatorname{Aut}\left(\mathbb{A}^{n}\right)$ on the vector fields $\operatorname{Vec}\left(\mathbb{A}^{n}\right)$ by the formula $\operatorname{Ad}(\varphi) \delta:=\varphi^{*-1} \circ \delta \circ \varphi^{*}$. In more geometric terms, considering $\delta$ as a section of the tangent bundle $T \mathbb{A}^{n}=\mathbb{A}^{n} \times K^{n} \rightarrow \mathbb{A}^{n}$, one defines the pull-back of $\delta$ by

$$
\varphi^{*}(\delta):=(d \varphi)^{-1} \circ \delta \circ \varphi, \quad \text { i.e., } \quad \varphi^{*}(\delta)_{a}=\left(d \varphi_{a}\right)^{-1}\left(\delta_{\varphi(a)}\right) \quad \text { for } a \in \mathbb{A}^{n} .
$$

Clearly, $\varphi^{*}(\delta)=\operatorname{Ad}\left(\varphi^{-1}\right) \delta$. However, the second formula above shows the well-known fact that the pull-back $\varphi^{*}(\delta)$ of a vector field $\delta$ is also defined for an étale morphism $\varphi: \mathbb{A}^{n} \rightarrow \mathbb{A}^{n}$. In the holomorphic setting this can be understood as lifting the corresponding integral curves.

Proposition 4.1. Let $\varphi: \mathbb{A}^{n} \rightarrow \mathbb{A}^{n}$ be an étale morphism. For any vector field $\delta \in$ $\operatorname{Vec}\left(\mathbb{A}^{n}\right)$ there is a vector field $\varphi^{*}(\delta) \in \operatorname{Vec}\left(\mathbb{A}^{n}\right)$ defined by $\varphi^{*}(\delta)_{a}:=(d \varphi)_{a}^{-1} \delta_{\varphi(a)}$ for $a \in \mathbb{A}^{n}$. It is uniquely determined by

$$
\varphi^{*}(\delta) \varphi^{*}(f)=\varphi^{*}(\delta f) \quad \text { for } f \in K\left[x_{1}, \ldots, x_{n}\right] .
$$

The map $\varphi^{*}: \operatorname{Vec}\left(\mathbb{A}^{n}\right) \rightarrow \operatorname{Vec}\left(\mathbb{A}^{n}\right)$ is an injective homomorphism of Lie algebras satisfying $\varphi^{*}(h \delta)=\varphi^{*}(h) \varphi^{*}(\delta)$ for $h \in K\left[x_{1}, \ldots, x_{n}\right]$. Moreover, $(\eta \circ \varphi)^{*}=\varphi^{*} \circ \eta^{*}$.

Proof. For a vector field $\delta: \mathbb{A}^{n} \rightarrow T \mathbb{A}^{n}$ and $a \in \mathbb{A}^{n}$ we have $(d \varphi \circ \delta)_{a}=d \varphi_{a}\left(\delta_{a}\right)$. Thus, the equation $(d \varphi)_{a}\left(\tilde{\delta}_{a}\right)=(\tilde{\delta} \circ \varphi)_{a}=\tilde{\delta}_{\varphi(a)}$ for the field $\tilde{\delta}$ has a unique solution, namely

$$
\tilde{\delta}_{a}:=\left(d \varphi_{a}\right)^{-1}\left(\delta_{\varphi(a)}\right)
$$

which is well-defined since $d \varphi_{a}$ is invertible. The Jacobian $\operatorname{determinant} \operatorname{det}(\operatorname{Jac}(\varphi))$ is a nonzero constant, and so the inverse matrix $\operatorname{Jac}(\varphi)^{-1}$ has entries in $K\left[x_{1}, \ldots, x_{n}\right]$. Therefore, the vector field $\varphi^{*}(\delta):=\tilde{\delta}$ defined above is polynomial, and it satisfies (4.1). This proves the first part of the proposition and shows that $\varphi^{*}$ is injective. Using (4.1) we find

$$
\varphi^{*}\left(\left(\delta_{1} \delta_{2}\right) f\right)=\varphi^{*}\left(\delta_{1}\left(\delta_{2} f\right)\right)=\varphi^{*}\left(\delta_{1}\right) \varphi^{*}\left(\delta_{2} f\right)=\left(\varphi^{*}\left(\delta_{1}\right) \varphi^{*}\left(\delta_{2}\right)\right) \varphi^{*}(f),
$$


hence $\varphi^{*}\left(\left[\delta_{1}, \delta_{2}\right] f\right)=\left[\varphi^{*}\left(\delta_{1}\right), \varphi^{*}\left(\delta_{2}\right)\right] \varphi^{*}(f)$, and so $\varphi^{*}\left(\left[\delta_{1}, \delta_{2}\right]\right)=\left[\varphi^{*}\left(\delta_{1}\right), \varphi^{*}\left(\delta_{2}\right)\right]$. Moreover,

$$
\varphi^{*}(h \delta) \varphi^{*}(f)=\varphi^{*}((h \delta) f)=\varphi^{*}(h) \varphi^{*}(\delta f)=\varphi^{*}(h) \varphi^{*}(\delta) \varphi^{*}(f),
$$

hence $\varphi^{*}(h \delta)=\varphi^{*}(h) \varphi^{*}(\delta)$. This proves the second part of the proposition, and the last claim is obvious.

Remark 4.2. In the notation of the proposition above let $\varphi=\left(f_{1}, \ldots, f_{n}\right)$. Then we get $\varphi^{*}\left(\delta x_{i}\right)=\varphi^{*}(\delta) f_{i}=\sum_{j} \frac{\partial f_{i}}{\partial x_{j}} \varphi^{*}(\delta) x_{j}$. Hence, for $\delta=\partial_{x_{k}}$, we obtain

$$
\delta_{i k}=\varphi^{*}\left(\partial_{x_{k}}\right) f_{i}=\sum_{j} \frac{\partial f_{i}}{\partial x_{j}} \varphi^{*}\left(\partial_{x_{k}}\right) x_{j} .
$$

This shows that the matrix $\left(\varphi^{*}\left(\partial_{x_{k}}\right) x_{j}\right)_{(j, k)}$ is invertible, $\left(\varphi^{*}\left(\partial_{x_{k}}\right) x_{j}\right)_{(j, k)}^{-1}=\operatorname{Jac}(\varphi)$, and

$$
\partial_{x_{i}}=\sum_{j} \frac{\partial f_{i}}{\partial x_{j}} \varphi^{*}\left(\partial_{x_{j}}\right) .
$$

Proposition 4.3. Let $\varphi: \mathbb{A}^{n} \rightarrow \mathbb{A}^{n}$ be an étale morphism. Then the pull-back map

$$
\varphi^{*}: \operatorname{Vec}\left(\mathbb{A}^{n}\right) \rightarrow \operatorname{Vec}\left(\mathbb{A}^{n}\right)
$$

is an isomorphism if and only if $\varphi$ is an automorphism.

Proof. Assume that $\varphi^{*}: \operatorname{Vec}\left(\mathbb{A}^{n}\right) \rightarrow \operatorname{Vec}\left(\mathbb{A}^{n}\right)$ is an isomorphism. Since $\varphi$ is étale, the comorphism $\varphi^{*}: K\left[x_{1}, \ldots, x_{n}\right] \rightarrow K\left[x_{1}, \ldots, x_{n}\right]$ is injective, and we only have to show that it is surjective. Proposition 4.1 implies that $\varphi^{*}\left(\operatorname{Vec}\left(\mathbb{A}^{n}\right)\right)=$ $\sum_{i} \varphi^{*}\left(K\left[x_{1}, \ldots, x_{n}\right]\right) \varphi^{*}\left(\partial_{x_{i}}\right)$, and from (4.2) we get

$$
\operatorname{Vec}\left(\mathbb{A}^{n}\right)=\bigoplus_{i} K\left[x_{1}, \ldots, x_{n}\right] \partial_{x_{i}}=\bigoplus_{i} K\left[x_{1}, \ldots, x_{n}\right] \varphi^{*}\left(\partial_{x_{i}}\right) .
$$

Hence $\varphi^{*}\left(\operatorname{Vec}\left(\mathbb{A}^{n}\right)\right)=\operatorname{Vec}\left(\mathbb{A}^{n}\right)$ if and only if $\varphi^{*}\left(K\left[x_{1}, \ldots, x_{n}\right]\right)=K\left[x_{1}, \ldots, x_{n}\right]$.

As a corollary of the two propositions above, we get the following result due to Kulikov [Ku192, Theorem 4].

Corollary 4.4. If every injective endomorphism of the Lie algebra $\operatorname{Vec}\left(\mathbb{A}^{n}\right)$ is an automorphism, then the Jacobian Conjecture holds in dimension $n$.

Remark 4.5. The result of Kulikov is stronger. He proves that every injective endomorphism of $\operatorname{Vec}\left(\mathbb{A}^{n}\right)$ is induced by an étale map $\varphi$, which also implies the converse of the statement above: If the Jacobian Conjecture holds in dimension $n$, then every injective endomorphism of $\operatorname{Vec}\left(\mathbb{A}^{n}\right)$ is an automorphism.

We finish this section by showing that if the divergence of a vector field is a constant, then the divergence is invariant under an étale morphism. More generally, we have the following result. 
Proposition 4.6. Let $\varphi: \mathbb{A}^{n} \rightarrow \mathbb{A}^{n}$ be an étale morphism, and let $\delta$ be a vector field. Then $\operatorname{Div} \varphi^{*}(\delta)=\varphi^{*}(\operatorname{Div} \delta)$. In particular, $\delta \in \operatorname{Vec}^{c}\left(\mathbb{A}^{n}\right)$ if and only if $\varphi^{*}(\delta) \in \operatorname{Vec}^{c}\left(\mathbb{A}^{n}\right)$, and in this case we have $\operatorname{Div} \varphi^{*}(\delta)=\operatorname{Div} \delta$.

Proof. Set $\varphi=\left(f_{1}, \ldots, f_{n}\right), \delta=\sum_{j} h_{j} \partial_{x_{j}}$ and $\varphi^{*}(\delta)=\sum_{j} \tilde{h}_{j} \partial_{x_{j}}$. Then, by (4.1),

$$
h_{k}\left(f_{1}, \ldots, f_{n}\right)=\sum_{i} \tilde{h}_{i} \frac{\partial f_{k}}{\partial x_{i}} \quad \text { for } k=1, \ldots, n
$$

Applying $\frac{\partial}{\partial x_{j}}$ to the left hand side we get the matrix

$$
\left(\sum_{i} \frac{\partial h_{k}}{\partial x_{i}}\left(f_{1}, \ldots, f_{n}\right) \frac{\partial f_{i}}{\partial x_{j}}\right)_{(k, j)}=H\left(f_{1}, \ldots, f_{n}\right) \cdot \operatorname{Jac}(\varphi)
$$

where $H:=\operatorname{Jac}\left(h_{1}, \ldots, h_{n}\right)$. On the right hand side, we obtain similarly

$$
\left(\sum_{i} \frac{\partial \tilde{h}_{i}}{\partial x_{j}} \frac{\partial f_{k}}{\partial x_{i}}+\sum_{i} \tilde{h}_{i} \frac{\partial^{2} f_{k}}{\partial x_{i} \partial x_{j}}\right)_{(k, j)}=\tilde{H} \cdot \operatorname{Jac}(\varphi)+\sum_{i} \tilde{h}_{i} \frac{\partial}{\partial x_{i}} \operatorname{Jac}(\varphi)
$$

Multiplying this matrix equation on the right by $\operatorname{Jac}(\varphi)^{-1}$ we finally get

$$
H\left(f_{1}, \ldots, f_{n}\right)=\tilde{H}+\sum_{i} \tilde{h}_{i} \frac{\partial}{\partial x_{i}} \operatorname{Jac}(\varphi) \cdot \operatorname{Jac}(\varphi)^{-1}
$$

Now we take traces on both sides. Using Lemma 4.7 below and the obvious equalities $\operatorname{Div} \delta=\operatorname{tr} H$ and $\operatorname{Div} \tilde{\delta}=\operatorname{tr} \tilde{H}$, we finally get

$$
\operatorname{Div} \tilde{\delta}=(\operatorname{Div} \delta)\left(f_{1}, \ldots, f_{n}\right)=\varphi^{*}(\operatorname{Div} \delta)
$$

The claim follows.

Lemma 4.7. Let $A$ be an $n \times n$ matrix whose entries $a_{i j}(t)$ are polynomials in $t$. Then

$$
\operatorname{tr}\left(\frac{d}{d t} A \cdot \operatorname{Adj}(A)\right)=\frac{d}{d t} \operatorname{det} A
$$

where $\operatorname{Adj}(A)$ is the adjoint matrix of $A$.

The proof is a nice exercise in linear algebra which we leave to the reader. It holds for rational entries $a_{i j}(t)$ over any field $K$, and in case $K=\mathbb{R}$ or $\mathbb{C}$ also for differentiable entries $a_{i j}(t)$. 


\section{Proof of the Main Theorem, part II}

We have seen that the canonical map $\operatorname{Ad}: \operatorname{Aut}\left(\mathbb{A}^{n}\right) \rightarrow \operatorname{Aut}_{\text {Lie }}\left(\operatorname{Vec}\left(\mathbb{A}^{n}\right)\right)$ is an isomorphism (Theorem 3.1). It follows from Proposition 4.6 that every automorphism of $\operatorname{Vec}\left(\mathbb{A}^{n}\right)$ induces an automorphism of $\operatorname{Vec}^{c}\left(\mathbb{A}^{n}\right)$. Moreover, since

$$
\operatorname{Vec}^{0}\left(\mathbb{A}^{n}\right)=\left[\operatorname{Vec}^{c}\left(\mathbb{A}^{n}\right), \operatorname{Vec}^{c}\left(\mathbb{A}^{n}\right)\right]
$$

(Lemma 1.3), we get a canonical map $\operatorname{Aut}_{\text {Lie }}\left(\operatorname{Vec}^{c}\left(\mathbb{A}^{n}\right)\right) \rightarrow \operatorname{Aut}_{\text {Lie }}\left(\operatorname{Vec}^{0}\left(\mathbb{A}^{n}\right)\right)$, which is easily seen to be injective. Thus the main theorem follows from the next result.

Theorem 5.1. The canonical map $\operatorname{Ad}: \operatorname{Aut}\left(\mathbb{A}^{n}\right) \rightarrow \operatorname{Aut}_{\mathrm{Lie}}\left(\operatorname{Vec}^{0}\left(\mathbb{A}^{n}\right)\right)$ is an isomorphism.

The proof needs some preparation. The next proposition is a reformulation of some results from [Now86] and [LD12]. For the convenience of the reader we will give a short proof.

Proposition 5.2. Let $\delta_{1}, \ldots, \delta_{n} \in \operatorname{Vec}\left(\mathbb{A}^{n}\right)$ be pairwise commuting and $K$-linearly independent vector fields. Then the following statements are equivalent:

(i) There is an étale morphism $\varphi: \mathbb{A}^{n} \rightarrow \mathbb{A}^{n}$ such that $\varphi^{*}\left(\partial_{x_{i}}\right)=\delta_{i}$ for all $i$.

(ii) $\operatorname{Vec}\left(\mathbb{A}^{n}\right)=\bigoplus_{i} K\left[x_{1}, \ldots, x_{n}\right] \delta_{i}$.

(iii) There exist $f_{1}, \ldots, f_{n} \in K\left[x_{1}, \ldots, x_{n}\right]$ such that $\delta_{i}\left(f_{j}\right)=\delta_{i j}$.

(iv) $\delta_{1}, \ldots, \delta_{n}$ do not have a common Darboux polynomial.

Recall that a common Darboux polynomial of the $\delta_{i}$ is a nonconstant polynomial $f \in$ $K\left[x_{1}, \ldots, x_{n}\right]$ such that $\delta_{i}(f)=h_{i} f$ for some $h_{i} \in K\left[x_{1}, \ldots, x_{n}\right], i=1, \ldots, n$.

Proof. (a) It follows from Remark 4.2 that (i) implies (ii) and (iii). Clearly, (ii) implies (iv) since a common Darboux polynomial for the $\delta_{i}$ is also a common Darboux polynomial for the $\partial_{x_{i}}$, which does not exist.

(b) We now show that (ii) implies (i), hence (iii), using the following well-known fact. If $h_{1}, \ldots, h_{n} \in K\left[x_{1}, \ldots, x_{n}\right]$ satisfy the conditions $\frac{\partial h_{i}}{\partial x_{j}}=\frac{\partial h_{j}}{\partial x_{i}}$ for all $i, j$, then there is an $f \in K\left[x_{1}, \ldots, x_{n}\right]$ such that $h_{i}=\frac{\partial f}{\partial x_{i}}$ for all $i$.

By (ii) we have $\partial_{x_{i}}=\sum_{k} h_{i k} \delta_{k}$ for $i=1, \ldots, n$. We claim that $\frac{\partial h_{i k}}{\partial x_{j}}=\frac{\partial h_{j k}}{\partial x_{i}}$ for all $i, j, k$. In fact,

$$
\begin{aligned}
0 & =\partial_{x_{i}} \partial_{x_{j}}-\partial_{x_{j}} \partial_{x_{i}}=\partial_{x_{i}} \sum_{k} h_{j k} \delta_{k}-\partial_{x_{j}} \sum_{k} h_{i k} \delta_{k} \\
& =\sum_{k} \frac{\partial h_{j k}}{\partial x_{i}} \delta_{k}+\sum_{k} h_{j k} \partial_{x_{i}} \delta_{k}-\sum_{k} \frac{\partial h_{i k}}{\partial x_{j}} \delta_{k}-\sum_{k} h_{i k} \partial_{x_{j}} \delta_{k} \\
& =\sum_{k}\left(\frac{\partial h_{j k}}{\partial x_{i}}-\frac{\partial h_{i k}}{\partial x_{j}}\right) \delta_{k}+\left(\sum_{k, \ell} h_{j k} h_{i \ell} \delta_{\ell} \delta_{k}-\sum_{k, \ell} h_{i k} h_{j \ell} \delta_{\ell} \delta_{k}\right) \\
& =\sum_{k}\left(\frac{\partial h_{j k}}{\partial x_{i}}-\frac{\partial h_{i k}}{\partial x_{j}}\right) \delta_{k}+\sum_{k, \ell} h_{i k} h_{j \ell}\left[\delta_{k}, \delta_{\ell}\right]=\sum_{k}\left(\frac{\partial h_{j k}}{\partial x_{i}}-\frac{\partial h_{i k}}{\partial x_{j}}\right) \delta_{k} .
\end{aligned}
$$


Hence $h_{i k}=\frac{\partial f_{k}}{\partial x_{i}}$ for suitable $f_{1}, \ldots, f_{n} \in K\left[x_{1}, \ldots, x_{n}\right]$. It is clear that the matrix $\left(h_{i k}\right)$ is invertible. This implies that the morphism $\varphi:=\left(f_{1}, \ldots, f_{n}\right): \mathbb{A}^{n} \rightarrow \mathbb{A}^{n}$ is étale, and $\partial_{x_{i}}=\sum_{k} \frac{\partial f_{k}}{\partial x_{i}} \delta_{k}$, hence $\delta_{k}=\varphi^{*}\left(\partial_{x_{k}}\right)$, by equation (4.2).

(c) Assume that (iii) holds. Setting $\delta_{i}=\sum_{k} h_{i k} \partial_{x_{k}}$ and applying both sides to $f_{j}$, we see that the matrix $\left(h_{i k}\right) \in \mathrm{M}_{n}\left(K\left[x_{1}, \ldots, x_{n}\right]\right)$ is invertible, hence (ii) holds. Thus the first three statements of the proposition are equivalent, and they imply (iv).

(d) Finally, assume that (iv) holds. Set $\delta_{i}=\sum_{k} h_{i k} \partial_{x_{k}}$. Since $\left[\delta_{i}, \delta_{j}\right]=0$ we get $\delta_{i}\left(h_{j k}\right)=\delta_{j}\left(h_{i k}\right)$ for all $i, j, k$. Now an easy calculation shows that $\delta_{k}\left(\operatorname{det}\left(h_{i j}\right)\right)=$ $\operatorname{Div}\left(\delta_{k}\right) \operatorname{det}\left(h_{i j}\right)$, and so $\operatorname{det}\left(h_{i j}\right) \in K$. If $\operatorname{det}\left(h_{i j}\right) \neq 0$, then (ii) follows.

If $\operatorname{det}\left(h_{i j}\right)=0$, then $\operatorname{rank}\left(\sum_{i=1}^{n} K\left[x_{1}, \ldots, x_{n}\right] \delta_{i}\right)=r<n$, and we can assume that $\operatorname{rank}\left(\sum_{i=1}^{r} K\left[x_{1}, \ldots, x_{n}\right] \delta_{i}\right)=r$. Choose a nontrivial relation $\sum_{i=1}^{r+1} f_{i} \delta_{i}=0$ where $\operatorname{gcd}\left(f_{1}, \ldots, f_{r+1}\right)=1$. Since $0=\delta_{j}\left(\sum_{i=1}^{r+1} f_{i} \delta_{i}\right)=\sum_{i=1}^{r+1} \delta_{j}\left(f_{i}\right) \delta_{i}$ for any $j$, we see that $\delta_{j}\left(f_{i}\right) \in K\left[x_{1}, \ldots, x_{n}\right] f_{i}$, and since the $\delta_{j}$ are $K$-linearly independent, at least one of the $f_{i}$ is not a constant, hence a common Darboux polynomial, contradicting (iv).

The second main ingredient for the proof is the following result.

Lemma 5.3. Let $\delta_{1}, \delta_{2} \in \operatorname{Vec}^{0}\left(\mathbb{A}^{n}\right)$ be commuting vector fields. Assume that:

(a) $\delta_{1}$ and $\delta_{2}$ have a common Darboux polynomial $f$ where $\delta_{i} f \neq 0, i=1,2$.

(b) Each $\delta_{i}$ acts locally nilpotently on $\operatorname{Vec}^{0}\left(\mathbb{A}^{n}\right)$.

Then $K\left[x_{1}, \ldots, x_{n}\right] \delta_{1}+K\left[x_{1}, \ldots, x_{n}\right] \delta_{2} \subseteq \operatorname{Vec}\left(\mathbb{A}^{n}\right)$ is a $K\left[x_{1}, \ldots, x_{n}\right]$-submodule of rank $\leq 1$.

Proof. We will show that there are nonzero polynomials $p_{1}, p_{2}$ such that $p_{1} \delta_{1}=p_{2} \delta_{2}$. We have $\delta_{i}(f)=h_{i} f$ where $h_{1}, h_{2} \neq 0$. Since $\delta_{1}$ and $\delta_{2}$ commute, we get $\delta_{1}\left(h_{2} f\right)=$ $\delta_{2}\left(h_{1} f\right)$, and so $\delta_{1} h_{2}=\delta_{2} h_{1}$. In view of the formula $\operatorname{Div}(g \delta)=\delta g+g \operatorname{Div}(\delta)$, this implies that $\delta:=h_{1} \delta_{2}-h_{2} \delta_{1} \in \operatorname{Vec}^{0}\left(\mathbb{A}^{n}\right)$. Moreover, $\delta f=0$, and so $f \delta \in \operatorname{Vec}^{0}\left(\mathbb{A}^{n}\right)$. Since

$$
\left[\delta_{1}, \xi\right]=\left[\delta_{1}, h_{1} \delta_{2}\right]-\left[\delta_{1}, h_{2} \delta_{1}\right]=\left(\delta_{1} h_{1}\right) \delta_{2}-\left(\delta_{1} h_{2}\right) \delta_{1},
$$

we get $\left(\operatorname{ad} \delta_{1}\right)^{k} \delta=\delta_{1}^{k}\left(h_{1}\right) \delta_{2}-\delta_{1}^{k}\left(h_{2}\right) \delta_{1}$ and $\left(\operatorname{ad} \delta_{1}\right)^{k}(f \delta)=\delta_{1}^{k}\left(f h_{1}\right) \delta_{2}-\delta_{1}^{k}\left(f h_{2}\right) \delta_{1}$. Now, by assumption (b), there is a $k>0$ such that $\left(\operatorname{ad} \delta_{1}\right)^{k} \delta=\left(\operatorname{ad} \delta_{1}\right)^{k}(f \delta)=0$, hence

$$
\delta_{1}^{k}\left(h_{1}\right) \delta_{2}=\delta_{1}^{k}\left(h_{2}\right) \delta_{1} \quad \text { and } \quad \delta_{1}^{k}\left(f h_{1}\right) \delta_{2}=\delta_{1}^{k}\left(f h_{2}\right) \delta_{1} .
$$

Thus the claim follows except if $\delta_{1}^{k} h_{1}=\delta_{1}^{k} h_{2}=\delta_{1}^{k}\left(f h_{1}\right)=\delta_{1}^{k}\left(f h_{2}\right)=0$. We will show that this leads to a contradiction. Since $\delta_{1} f=h_{1} f$, we get $\delta_{1}^{k+1} f=0$. Choose $r, s$ minimal with $\delta_{1}^{r} h_{1}=0$ and $\delta_{1}^{s} f=0$. By assumption, $r, s \geq 1$, and we get $\delta_{1}^{r+s-2}\left(h_{1} f\right)=$ $\delta_{1}^{r-1} h_{1} \cdot \delta_{1}^{s-1} f \neq 0$. On the other hand, $\delta_{1}^{s-1}\left(h_{1} f\right)=\delta_{1}^{s} f=0$, and we end up with a contradiction, because $s-1 \leq r+s-2$.

Now we can prove the Theorem.

Proof of Theorem 5.1. The case $n=1$ is handled in Lemma 3.2, so we can assume that $n \geq 2$. Let $\theta$ be an automorphism of $\operatorname{Vec}^{0}\left(\mathbb{A}^{n}\right)$ as a Lie algebra, and set $\delta_{i}:=\theta\left(\partial_{x_{i}}\right)$. Then the vector fields $\delta_{1}, \ldots, \delta_{n}$ are pairwise commuting and $K$-linearly independent. Since 
$\partial_{x_{i}}$ acts locally nilpotently on $\operatorname{Vec}^{0}\left(\mathbb{A}^{n}\right)$, the same holds for $\delta_{i}$. Moreover, the centralizer of the $\delta_{i}$ in $\operatorname{Vec}^{0}\left(\mathbb{A}^{n}\right)$ is the linear span of the $\delta_{i}$, i.e. $\left[\delta, \delta_{i}\right]=0$ for all $i$ implies that $\delta \in \bigoplus_{i} K \delta_{i}$. In the following we will use vector fields with rational coefficients:

$$
\operatorname{Vec}{ }^{\mathrm{rat}}\left(\mathbb{A}^{n}\right):=K\left(x_{1}, \ldots, x_{n}\right) \otimes_{K\left[x_{1}, \ldots, x_{n}\right]} \operatorname{Vec}\left(\mathbb{A}^{n}\right)=\bigoplus_{i=1}^{n} K\left(x_{1}, \ldots, x_{n}\right) \partial_{x_{i}} .
$$

(1) We first claim that the $\delta_{i}$ do not have a common Darboux polynomial. So assume that there exists a nonconstant $f \in K\left[x_{1}, \ldots, x_{n}\right]$ such that $\delta_{i} f=h_{i} f$ for all $i$ and some $h_{i} \in K\left[x_{1}, \ldots, x_{n}\right]$.

First assume that $h_{1}=0$, i.e. $\delta_{1} f=0$. Then $f \delta_{1} \in \operatorname{Vec}^{0}\left(\mathbb{A}^{n}\right)$, and for any $h \in$ $K\left[x_{1}, \ldots, x_{n}\right]$ and every $i$ we have $\left[\delta_{i}, h f \delta_{1}\right]=\delta_{i}(h f) \delta_{1}=\left(\delta_{i}(h)+h h_{i}\right) f \delta_{1}$, and so

$$
\left(\operatorname{ad} \delta_{i}\right)^{k}\left(K\left[x_{1}, \ldots, x_{n}\right] f \delta_{1}\right) \subseteq K\left[x_{1}, \ldots, x_{n}\right] f \delta_{1} \quad \text { for all } k \geq 0 .
$$

Set $\eta:=\theta^{-1}\left(f \delta_{1}\right)$. Then there are $k_{1}, \ldots, k_{n} \in \mathbb{N}$ such that

$$
\eta_{0}:=\left(\operatorname{ad} \partial_{x_{1}}\right)^{k_{1}}\left(\operatorname{ad} \partial_{x_{2}}\right)^{k_{2}} \cdots\left(\operatorname{ad} \partial_{x_{n}}\right)^{k_{n}} \eta \in K \partial_{x_{1}} \oplus \cdots \oplus K \partial_{x_{n}} \backslash\{0\} .
$$

Hence, $\theta\left(\eta_{0}\right)=\left(\operatorname{ad} \delta_{1}\right)^{k_{1}}\left(\operatorname{ad} \delta_{2}\right)^{k_{2}} \cdots\left(\operatorname{ad} \delta_{n}\right)^{k_{n}}\left(f \delta_{1}\right) \in K \delta_{1} \oplus \cdots \oplus K \delta_{n} \backslash\{0\}$, which contradicts (5.1), because $f \notin K$.

We are left with the case where no $h_{i}$ is zero. Then Lemma 5.3 above implies that $\sum_{i} K\left[x_{1}, \ldots, x_{n}\right] \delta_{i} \subseteq \operatorname{Vec}\left(\mathbb{A}^{n}\right)$ has rank 1, i.e. there exist $\delta \in \operatorname{Vec}\left(\mathbb{A}^{n}\right)$ and nonzero rational functions $r_{i} \in K\left(x_{1}, \ldots, x_{n}\right)$ such that $\delta_{i}=r_{i} \delta$ for $i=1, \ldots, n$. We can assume that $\delta$ is minimal, i.e., not of the form $q \delta^{\prime}$ with a nonconstant polynomial $q$. For every $\mu$ commuting with $\delta_{i}$, we get $0=\left[\mu, \delta_{i}\right]=\left[\mu, r_{i} \delta\right]=\mu\left(r_{i}\right) \delta+r_{i}[\mu, \delta]$, hence $[\mu, \delta] \in K\left(x_{1}, \ldots, x_{n}\right) \delta$. It is easy to see that

$$
L:=\left\{\xi \in \operatorname{Vec}\left(\mathbb{A}^{n}\right) \mid[\xi, \delta] \in K\left(x_{1}, \ldots, x_{n}\right) \delta\right\}
$$

is a Lie subalgebra of $\operatorname{Vec}\left(\mathbb{A}^{n}\right)$ which contains all elements commuting with one of the $\delta_{i}$. Since $\operatorname{Vec}^{0}\left(\mathbb{A}^{n}\right)$ is generated, as a Lie algebra, by elements commuting with one of the $\partial_{x_{i}}$ we see that $\theta\left(\operatorname{Vec}^{0}\left(\mathbb{A}^{n}\right)\right)=\operatorname{Vec}^{0}\left(\mathbb{A}^{n}\right)$ is generated by the elements commuting with one of the $\delta_{i}$. Thus $\operatorname{Vec}^{0}\left(\mathbb{A}^{n}\right) \subseteq L$, and so $\left[\operatorname{Vec}^{0}\left(\mathbb{A}^{n}\right), \delta\right] \subseteq K\left(x_{1}, \ldots, x_{n}\right) \delta$. For $\delta=$ $\sum_{i} p_{i} \partial_{x_{i}}$ we get $\left[\partial_{x_{k}}, \delta\right]=\sum_{i} \frac{\partial}{\partial x_{k}} \partial_{x_{i}}=s \delta$ for some $s \in K\left(x_{1}, \ldots, x_{n}\right)$, hence $\frac{\partial p_{i}}{\partial x_{k}} p_{j}=$ $\frac{\partial p_{j}}{\partial x_{k}} p_{i}$ for all pairs $i, j$. This implies that $\frac{\partial}{\partial x_{k}} \frac{p_{j}}{p_{i}}=0$ in case $p_{i} \neq 0$, i.e. $\frac{p_{j}}{p_{i}}$ does not depend on $x_{k}$. Since this holds for all $k$, we conclude that $p_{j}=c_{j} p_{i}$ for some $c_{j} \in K$, hence $\delta=\sum_{j} c_{j} \partial_{x_{j}}$, because $\delta$ is minimal. In particular, $\left[\partial_{x_{k}}, \delta\right]=0$ for all $k$. Now we get $\left[x_{\ell} \partial_{x_{k}}, \delta\right]=-c_{\ell} \partial_{x_{k}} \in K\left(x_{1}, \ldots, x_{n}\right) \delta$ for all $k, \ell$, which implies $\delta=0$, hence a contradiction.

(2) Now we use the implication (vi) $\Rightarrow$ (i) of Proposition 5.2 to see that there is an étale morphism $\varphi: \mathbb{A}^{n} \rightarrow \mathbb{A}^{n}$ with $\delta_{i}=\varphi^{*}\left(\partial_{x_{i}}\right)$ for all $i$. Then the composition $\theta^{\prime}:=$ $\theta^{-1} \circ \varphi^{*}: \operatorname{Vec}^{0}\left(\mathbb{A}^{n}\right) \rightarrow \operatorname{Vec}^{0}\left(\mathbb{A}^{n}\right)$ is an injective homomorphism of Lie algebras (Proposition 4.1) and $\theta^{\prime}\left(\partial_{x_{i}}\right)=\partial_{x_{i}}$. Hence, Lemma 5.4 below implies that $\theta^{\prime}=\operatorname{Ad}(s)=\left(s^{-1}\right)^{*}$ where $s \in \operatorname{Aut}\left(\mathbb{A}^{n}\right)$ is a translation, hence $\theta=(\varphi \circ s)^{*}$. Now Proposition 4.3 implies that $\psi:=\varphi \circ s$ is an automorphism of $\mathbb{A}^{n}$, and so $\theta=\operatorname{Ad}\left(\psi^{-1}\right)$ as claimed. 
Lemma 5.4. Let $\theta$ be an injective endomorphism of $\operatorname{Vec}^{0}\left(\mathbb{A}^{n}\right)$ such that $\theta\left(\partial_{x_{i}}\right)=\partial_{x_{i}}$ for all $i$. Then $\theta=\operatorname{Ad}(s)$ where $s: \mathbb{A}^{n} \stackrel{\sim}{\rightarrow} \mathbb{A}^{n}$ is a translation. In particular, $\theta$ is an automorphism.

Proof. We know that $\sum_{i} K \partial_{x_{i}}=L(S)$ where $S \subset \operatorname{Aff}_{n}$ are the translations. Moreover, $L\left(\operatorname{Aff}_{n}\right)$ is the normalizer of $L(S)$ in the Lie algebra $\operatorname{Vec}\left(\mathbb{A}^{n}\right)$. Hence $\theta\left(L\left(\operatorname{SAff}_{n}\right)\right)=$ $L\left(\mathrm{SAff}_{n}\right)$, and so there is an affine transformation $g$ such that $\left.\operatorname{Ad}(g)\right|_{L\left(\mathrm{SAff}_{n}\right)}=\left.\theta\right|_{L\left(\mathrm{SAff}_{n}\right)}$, by Lemma 3.2. Since $\operatorname{Ad}(g)$ is the identity on $L(S)$, we see that $g$ is a translation. It follows that $\operatorname{Ad}\left(g^{-1}\right) \circ \theta$ is the identity on $L\left(\mathrm{SL}_{n}\right)$, hence $\operatorname{Ad}\left(g^{-1}\right) \circ \theta=\operatorname{Ad}(\lambda E)$ for some $\lambda \in K^{*}$, by Lemma 3.3. But $\lambda=1$, because $\theta$ is the identity on $L(S)$, and so $\theta=\operatorname{Ad}(g)$.

Acknowledgments. We thank the referee for pointing out a mistake in the proof Theorem 5.1.

The authors are partially supported by the SNF (Schweizerischer Nationalfonds).

\section{References}

[Bav13] Bavula, V. V.: The group of automorphisms of the Lie algebra of derivations of a polynomial algebra. J. Algebra Appl. 16 (2017), online, 8 pp.

[BKY12] Belov-Kanel, A., Yu, J.-T.: On the Zariski topology of automorphism groups of affine spaces and algebras. arXiv:1207.2045v1 (2012)

[Bor91] Borel, A.: Linear Algebraic Groups. 2nd ed., Grad. Texts in Math. 126, Springer, New York (1991) Zbl 0726.20030 MR 1102012

[Jor78] Jordan, D. A.: Simple Lie rings of derivations of commutative rings. J. London Math. Soc. (2) 18, 443-448 (1978) Zbl 0404.17009 MR 0506506

[Kra11] Kraft, H.: Algebraic transformation groups: An introduction. Mathematisches Institut, Universität Basel, http://www.math.unibas.ch/kraft (2014)

[Kra15] Kraft, H.: Automorphism groups of affine varieties and a characterization of affine $n$-space. arXiv:1501.06362 (2015)

[Kul92] Kulikov, V. S.: Generalized and local Jacobian problems. Izv. Ross. Akad. Nauk Ser. Mat. 56, 1086-1103 (1992) (in Russian) Zbl 0796.14008 MR 1209034

[Kum02] Kumar, S.: Kac-Moody Groups, Their Flag Varieties and Representation Theory. Progr. Math. 204, Birkhäuser Boston, Boston, MA (2002) Zbl 1026.17030 MR 1923198

[LD12] Li, J., Du, X.: Pairwise commuting derivations of polynomial rings. Linear Algebra Appl. 436, 2375-2379 (2012) Zbl 1236.13022

[Now86] Nowicki, A.: Commutative bases of derivations in polynomial and power series rings. J. Pure Appl. Algebra 40, 275-279 (1986) Zbl 0592.13004 MR 0836653

[Pro07] Procesi, C.: Lie Groups: An Approach through Invariants and Representations. Universitext, Springer, New York (2007) Zbl 1154.22001 MR 2265844

[Reg13] Regeta, A.: Lie subalgebras of vector fields and the Jacobian conjecture. arXiv:1311.0232 (2013)

[Sha66] Shafarevich, I. R.: On some infinite-dimensional groups. Rend. Mat. Appl. (5) 25, 208212 (1966) Zbl 0149.39003 MR 0485898

[Sha81] Shafarevich, I. R.: On some infinite-dimensional groups. II. Izv. Akad. Nauk SSSR Ser. Mat. 45, 214-226 (1981) (in Russian) Zbl 0475.14036 MR 1347084 